\title{
Severe congenital cutis laxa with cardiovascular manifestations due to homozygous deletions in ALDH18A1
}

\author{
Björn Fischer ${ }^{\mathrm{a}, \mathrm{b}}$, Bert Callewaert ${ }^{\mathrm{c}}$, Phillipe Schröter ${ }^{\mathrm{a}}$, Paul J. Coucke ${ }^{\mathrm{c}}$, Claire Schlack ${ }^{\mathrm{a}}$, Claus-Eric Ott ${ }^{\mathrm{a}, \mathrm{b}}$, \\ Manrico Morroni ${ }^{\mathrm{d}}$, Wolfgang Homann ${ }^{\mathrm{e}}$, Stefan Mundlos ${ }^{\mathrm{a}, \mathrm{b}, \mathrm{f}}$, Eva Morava ${ }^{\mathrm{g}}$, Anna Ficcadenti ${ }^{\mathrm{h}}$, Uwe Kornak ${ }^{\mathrm{a}, \mathrm{b}, \mathrm{f}, *}$ \\ a Institut fuer Medizinische Genetik und Humangenetik, Charité-Universitaetsmedizin Berlin, Augustenburger Platz 1, 13353 Berlin, Germany \\ b Max-Planck-Institut fuer Molekulare Genetik, FG Development E' Disease, Ihnestr. 63-73, 14195 Berlin, Germany \\ c Center for Medical Genetics, Ghent University Hospital, Ghent, Belgium

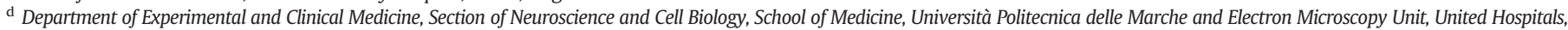 \\ Ancona, Italy \\ e Neonatologie, Christliches Kinderhospital Osnabrück, Osnabrück, Germany \\ ${ }^{\mathrm{f}}$ Berlin-Brandenburg Center for Regenerative Therapies, Charité-Universitaetsmedizin Berlin, Germany \\ g Tulane University Medical Center, Hayward Genetics Center, New Orleans, LA, USA \\ ${ }^{\mathrm{h}}$ Rare diseases Regional Centre, Pediatric Institute of Maternal-Infantile Sciences Department, Polytechnic University of Marche, Salesi Hospital of United Hospitals of Ancona, Italy
}

\section{A R T I C L E I N F O}

\section{Article history:}

Received 18 March 2014

Received in revised form 9 May 2014

Accepted 10 May 2014

Available online 21 May 2014

\section{Keywords:}

Autosomal recessive cutis laxa

ALDH18A1

Homozygous deletion 10q24.3

Progeroid features

PYCR1

Mitochondria

\begin{abstract}
A B S T R A C T
Autosomal recessive cutis laxa (ARCL) type 2 constitutes a heterogeneous group of diseases mainly characterized by lax and wrinkled skin, skeletal anomalies, and a variable degree of intellectual disability. ALDH18A1-related ARCL is the most severe form within this disease spectrum. Here we report on the clinical and molecular findings of two affected individuals from two unrelated families. The patients presented with typical features of de Barsy syndrome and an overall progeroid appearance. However, the phenotype was highly variable including cardiovascular involvement in the more severe case. Investigation of a skin biopsy of one patient revealed not only the typical alterations of elastic fibers, but also an altered structure of mitochondria in cutaneous fibroblasts. Using conventional sequencing and copy number analysis we identified a frameshift deletion of one nucleotide and a microdeletion affecting the ALDH18A1 gene, respectively, in a homozygous state in both patients. Expression analysis in dermal fibroblasts from the patient carrying the microdeletion showed an almost complete absence of the ALDH18A1 mRNA resulting in an absence of the ALDH18A1 protein. So far, only 13 affected individuals from seven unrelated families suffering from ALDH18A1-related cutis laxa have been described in literature. Our findings provide new insights into the clinical spectrum and show that beside point mutations microdeletions are a possible cause of ALDH18A1-ARCL.
\end{abstract}

(C) 2014 Elsevier Inc. All rights reserved.

\section{Introduction}

Syndromes with cutis laxa $(\mathrm{CL})$ are a heterogeneous group of diseases mainly characterized by lax and wrinkled skin, generalized connective tissue weakness, pulmonary and cardiovascular involvement, low bone mass, and a variable degree of intellectual disability. These conditions can either be inherited in an X-chromosomal, autosomal dominant (AD) or autosomal recessive (AR) fashion. The recessive forms show the most variable phenotypes and causative genetic defects. They can be subdivided into three distinct groups. AR cutis laxa type 1 (ARCL1; OMIM 219100) is associated with marked cardiovascular and/or pulmonary defects. Alterations in fibulin-5 (FBLN5) and latent transforming

\footnotetext{
* Corresponding author at: Institut fuer Medizinische Genetik und Humangenetik, Charité-Universitaetsmedizin Berlin, Augustenburger Platz 1, 13353 Berlin, Germany. E-mail address: uwe.kornak@charite.de (U. Kornak).
}

growth factor beta binding protein 4 (LTBP4) cause clinically resembling cutis laxa syndromes with severe pulmonary emphysema and gastrointestinal and genitourinary abnormalities [1,2]. Mutations in fibulin-4 (FBLN4, also known as EGF-containing fibulin-like extracellular matrix protein 2) were shown to cause a severe CL phenotype with serious cardiovascular problems including arterial tortuosity and aneurysm formation [2,3]. Another form with severe cardiovascular involvement, but often mild cutis laxa symptoms, is the arterial tortuosity syndrome (ATS; OMIM 208050) due to mutations in the SLC2A10 gene encoding a facilitated glucose transporter $[1,4]$. Autosomal recessive cutis laxa type 2, Debré type (ARCL2A; OMIM 219200) is caused by mutations in the ATP6V0A2 gene which encodes the a2 subunit of the V-ATPase localizing to the Golgi apparatus. This subtype is characterized by fine skin wrinkles, downslanting palpebral fissures, delayed fontanel closure and variable cortical brain anomalies [5,6]. MACS syndrome (Macrocephaly, Alopecia, Cutis laxa, and Scoliosis; OMIM 613075) is secondary to mutations in RIN2 encoding a component of the endosomal compartment which 
interacts with the small GTPase RAB5 [7]. Gerodermia osteodysplastica (GO; OMIM 231070), due to mutations in the GORAB gene, shows relatively mild cutis laxa, but a prematurely aged aspect and severe osteoporosis. GO belongs to ARCL type 2 . The GORAB protein is an interaction partner of the small GTPase RAB6 and, similar to ATP6V0A2 and RIN2, very likely involved in intracellular transport [8].

ARCL3 (DBS; OMIM 219150) refers to de Barsy syndrome, the most severe disease of the autosomal recessive cutis laxa spectrum. These patients show thin and translucent skin, visibility of the veins and a specific facial gestalt with triangular face and prognathism resulting in a progeroid appearance. Additionally, affected individuals show skeletal and ophthalmological abnormalities, intrauterine growth retardation and in the majority of cases considerable intellectual disability $[9,10]$. ARCL patients with de Barsy-like symptoms may harbor mutations in either PYCR1 (pyrollin-5-carboxylate reductase 1) (ARCLIIB; OMIM 612940 ) or ALDH18A1 (P5CS) (pyrrolin-5-carboxylate synthase 1) (ARCLIIIA; OMIM 219150). Both gene products are metabolic enzymes involved in the de novo biosynthesis of proline and were shown to be associated to mitochondria $[11,12]$.

\section{Material and methods}

\subsection{Patients}

In this study we report on two affected individuals from two unrelated families which fulfilled the major diagnostic criteria for de Barsy-like CL or PYCR1- and ALDH18A1-related ARCL. These criteria included: generalized skin wrinkling, intrauterine growth retardation, a typical facial gestalt, variable CNS involvement and corneal clouding or cataract. From patient 1 , a skin biopsy was taken and amino acid profiles were analyzed from plasma according to standard procedures. In both families DNA was isolated from peripheral blood samples.

\subsection{Mutation screening}

Sequencing of all exons and the flanking intron regions of the PYCR1 (NM_006907.2) and ALDH18A1 (NM_002860.3) genes was performed in the affected individual using BigDye Terminator cycle sequencing kit (Applied Biosystems) and run on a ABI 3730 DNA Analyzer (Applied Biosystems). Copy number analyses on genomic DNA were performed on ABI Prism 7900HT System as described previously [13]. All primer sequences are available on request. The molecular diagnostic tests were performed in an accredited laboratory (No.: DAP-ML-3869.00 (ISO 15189:2003 and ISO/IEC 17025:2005)).

\subsection{Cell culture}

Skin fibroblasts from patient 1 and from control individuals were cultivated in DMEM (Lonza) supplemented with $10 \%$ fetal calf serum (Gibco), 1\% ultraglutamine (Lonza) and 1\% penicillin/streptomycin (Lonza).

\subsection{Transmission electron microscopy}

Skin biopsy (tissue) from patient 1 was fixed in $2 \%$ glutaraldehyde/ $2 \%$ paraformaldehyde in $0.1 \mathrm{M}$ phosphate buffer for $3 \mathrm{~h}$ at $4{ }^{\circ} \mathrm{C}$, postfixed in $1 \%$ buffered osmium tetroxide, dehydrated in graded alcohols, and embedded in an Epon-Araldite mixture. Semithin sections $(2 \mu \mathrm{m})$ were obtained with a MICROM HM 355 microtome (Zeiss, Oberkochen, Germany) and stained with toluidine blue. Thin sections were obtained using an MTX ultramicrotome (RMC, Tucson, AZ, USA), stained with lead citrate and examined with a CM10 transmission electron microscope (Philips, Eindhoven, The Netherlands).

\section{5. mRNA expression-analysis}

Cells were lysed with Trizol (Invitrogen) and total RNA was prepared by a standard RNA extraction protocol. Total cDNA was reverse transcribed by RevertAid H Minus First Strand cDNA Synthesis Kit (Fermentas). Quantitative PCR was performed using EVAgreen (Solis BioDyne) on an ABI Prism 7500 (Applied Biosystems, Foster City, US). All primer sequences are available on request.

\subsection{Immunoblot}

Cells were lysed in RIPA buffer ( $150 \mathrm{mM} \mathrm{NaCl}, 50 \mathrm{mM}$ Tris, $5 \mathrm{mM}$ EDTA, 1\% Triton X-100, 0.25\% desoxycholate, 0.1\% SDS) supplemented with complete proteinase inhibitor (Roche). $10 \mu \mathrm{g}$ of protein per lane were separated by $10 \%$ SDS-PAGE. After blotting overnight, membranes were blocked and probed with antibodies against ALDH18A1 (Novus: NBP1-83324, Sigma: HPA008333 and Abcam: ab111977) or GAPDH (Ambion). Membranes were washed and incubated with HRPconjugated secondary antibodies and signals were detected using ECL reagent (Perkin Elmer). All blots were performed at least three times using different cell lysates.

\section{Results}

\subsection{Clinical presentation}

\subsubsection{Patient 1}

This 18 month old girl was the first child from a consanguineous couple (parents are first degree cousins) of Tunisian origin (Fig. 1A). She showed intrauterine growth retardation (weight: $1770 \mathrm{~g}(<3$ rd percentile); length: $39 \mathrm{~cm}$ ( $<3 \mathrm{rd}$ percentile); OFC: $31 \mathrm{~cm}(<3 \mathrm{rd}$ percentile)) and failure to thrive. Her psychomotor development was retarded and she developed intellectual disability and learning problems. No athetoid movements were recognized but she presented with disseminated tremors. On MRI no structural brain abnormalities were detectable. She showed craniofacial dysmorphism consisting of a typical triangular face, malar hypoplasia, prognathism and prominent ears leading to a prematurely aged appearance. Furthermore, she showed a mild cataract. A classical connective tissue phenotype with thin, lax and wrinkled skin at the abdomen and the dorsum of the hands and feet and visibility of veins was observed (Fig. 1C). She had finger contractures and furthermore showed hand and foot malformations. The middle phalanx of the fifth finger was hypoplastic on the right and absent on the left while the distal phalanx was normal bilaterally. Furthermore, the first metacarpal was bowed bilaterally (Fig. 1E). Additionally the proximal phalanx of the first toe was hypoplastic and she had a unilateral hip dislocation (Fig. $1 F$ ). The spine showed a dorsal hump due to a marked kyphosis (Fig. 1G). Further features were hypotonia, bilateral hearing loss and cardiovascular problems. A ventricular septal defect, atrial septal defect (ostium secundum type), patent ductus arteriosus and a persistence of superior left vena cava were recognized. Analysis of serum amino acids revealed low citrulline and arginine levels (Table 1 ). Additionally urinary organic acids, oligosaccharides, purines and pyrimidines were normal (data not shown). We performed a skin biopsy and analyzed the morphology of dermal extracellular matrix and fibroblasts by transmission electron microscopy. This investigation revealed alterations in the number and shape of elastic fibers typical for cutis laxa syndromes (Figs. 2A-B). Furthermore we found swollen mitochondria in $14 \%$ out of 139 fibroblasts analyzed (Figs. 2C-D).

\subsubsection{Patient 2}

This male patient originated from a consanguineous Turkish couple (first degree cousins) that previously had three healthy daughters and three early abortions (Fig. 1B). The pregnancy was complicated by maternal gestational diabetes necessitating insulin therapy. Prenatal ultrasounds indicated a single umbilical artery, rather short ribs and long 
A

Family 1
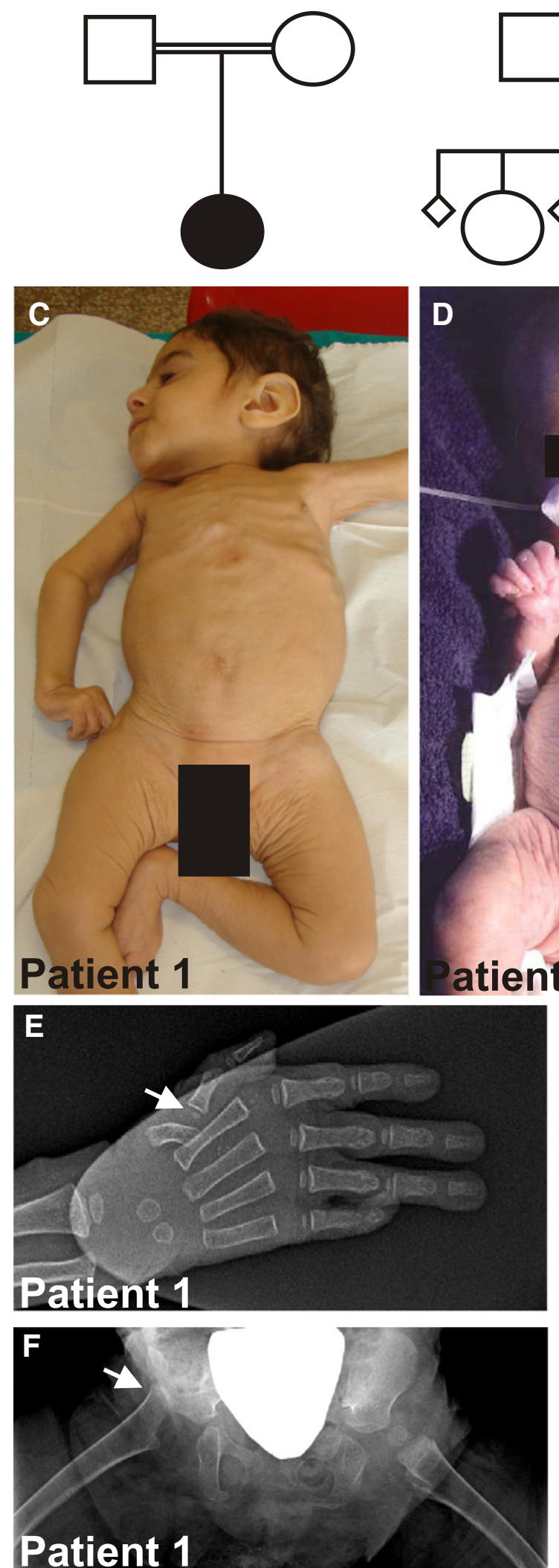

B Family 2
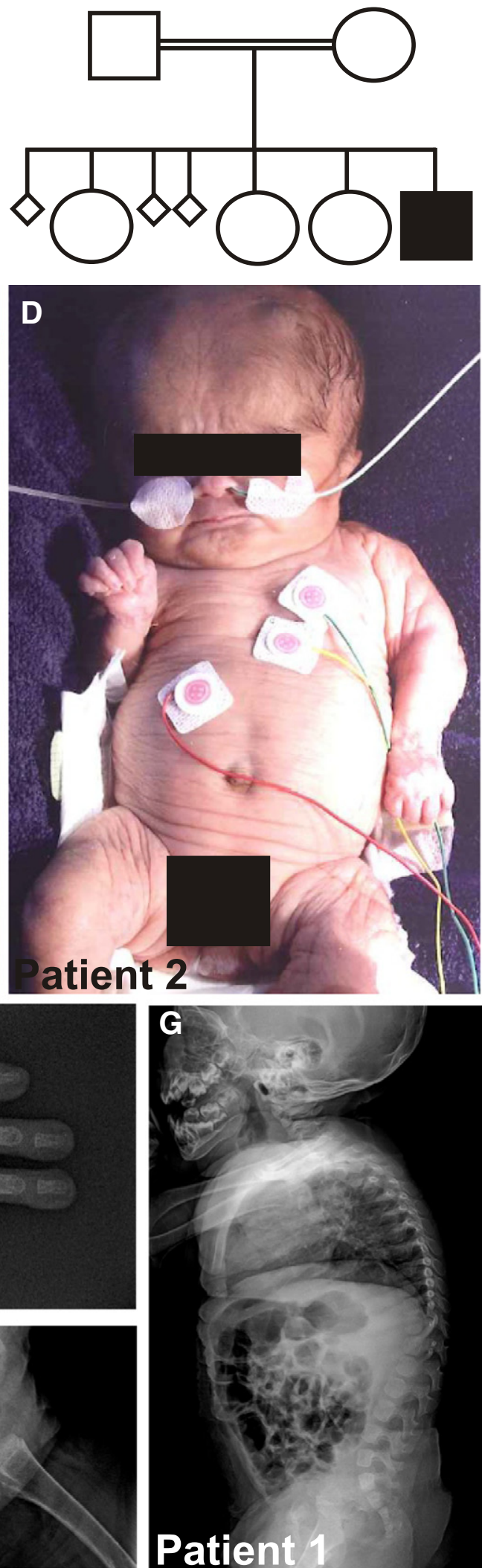

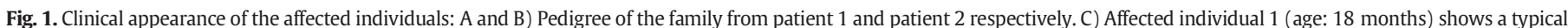

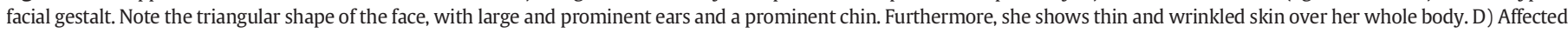

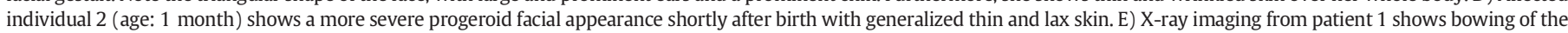
first metacarpal, F) a unilateral hip dislocation (arrowhead) and a missing middle phalanx of the fifth finger. G) The spine displays a severe kyphosis. 
Table 1

Selected plasma amino acid concentration in patient 1 .

\begin{tabular}{lcl}
\hline Amino acid & Plasma & \\
\cline { 2 - 3 } & Patient $\mu \mathrm{mol} / \mathrm{l}$ & Reference range $\mu \mathrm{mol} / \mathrm{l}$ \\
\hline Ornithine & 23 & $21-77$ \\
Citrulline & 3 & $27-43$ \\
Arginine & 39 & $49-129$ \\
Proline & 105 & $71-303$ \\
Glutamate & 61 & $8-64$ \\
Aspartate & 8 & $<14$ \\
\hline
\end{tabular}

bones, as well as a possible aortic stenosis. The proband was born at 35 weeks of gestation. He had intrauterine growth restriction birth length: $38 \mathrm{~cm}$ (<3rd percentile), birth weight $1370 \mathrm{~g}$ (<3rd percentile) and microcephaly (OFC at birth was $29.5 \mathrm{~cm}$ (3rd-10th percentile)). APGAR scores were 5, 9, and 9 after 1,5 , and $10 \mathrm{~min}$, respectively. The patient was intubated because of insufficient respiratory efforts. He showed agenesis of the corpus callosum, presented with seizures, and had a typical facial gestalt, prominent ears and corneal clouding leading to a prematurely aged appearance (Fig. 1D). He had a remarkable thin, translucent, lax and wrinkled skin throughout the body with clearly visible veins (Fig. 1D). He showed joint hypermobility and had shortening of the extremities, and adducted thumbs and the fingers II and V crossed fingers III and IV. Additionally, he showed congenital luxation of the left hip. Postnatal investigations confirmed a rather narrow aortic arch without a clear stenosis, short ribs and long bones, and a dilated pyelon of the right kidney. The neonatal course was further complicated by an intraventricular bleeding, grade III, probably secondary to an aneurysm near the cranial end of the arteria basilaris, progressive respiratory failure and a urosepsis causing death at 3 months of age. Unfortunately, no plasma, fresh blood or a fibroblast sample was available from this affected individual, nor was an autopsy performed to evaluate pulmonary emphysema.

\subsection{Identification of the causative genetic defect}

Due to the high frequency of PYCR1 mutations in our cohort of $\mathrm{CL}$ affected individuals, we first sequenced the coding region of this gene in both affected individuals without detecting any mutation. Subsequently, we amplified the coding exons 2 to 18 of the ALDH18A1 gene in both affected individuals.

In patient 1 amplification of ALDH18A1 exon 15 repeatedly revealed no product, whereas all other exons yielded PCR products. The amplified products were sequenced and showed no pathogenic alterations. To investigate whether a suspected microdeletion of exon 15 in the ALDH18A1 gene is the cause of the disease we performed copy number analysis by qPCR. The amplicons representing exons 14 and 16 showed normal signal intensities in all individuals analyzed. For the exon 15 amplicon we observed a dramatic reduction in signal intensity in the DNA from the affected individual suggesting a homozygous deletion. The parents showed an approximately 50\% reduction as expected for

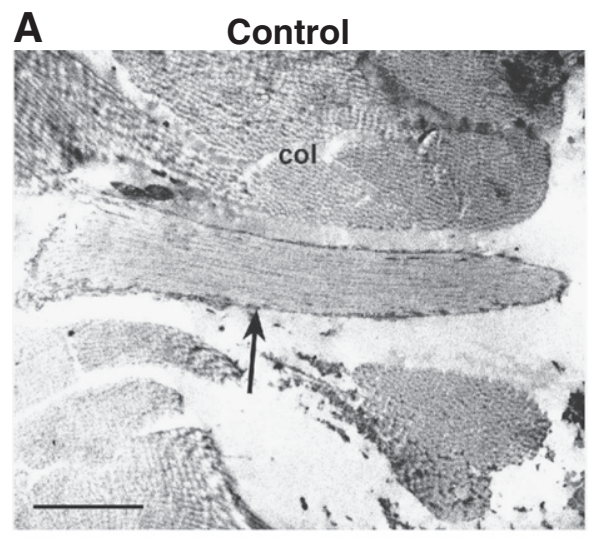

B

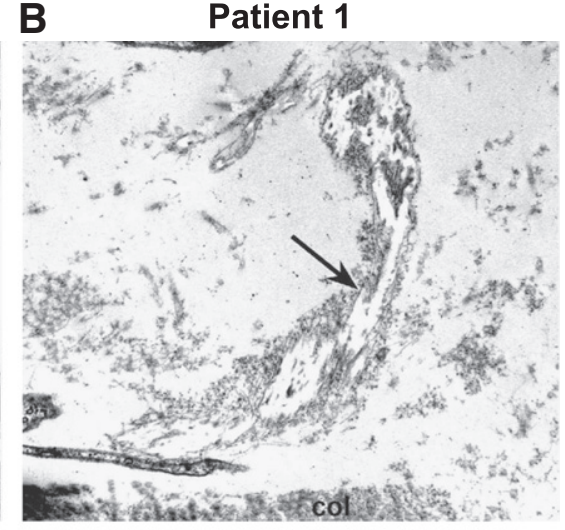

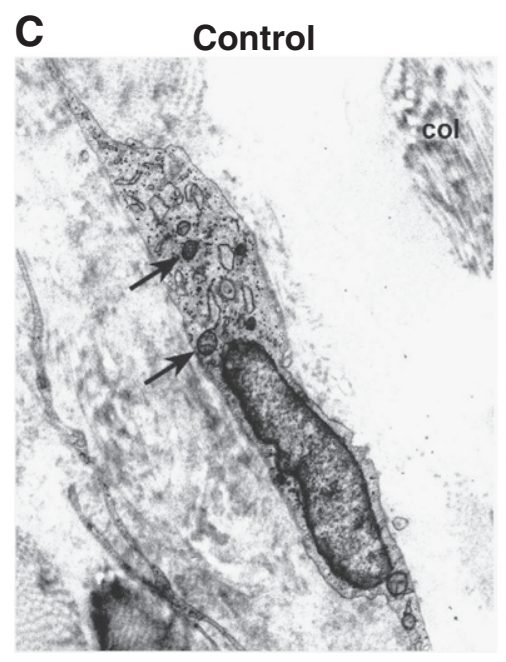

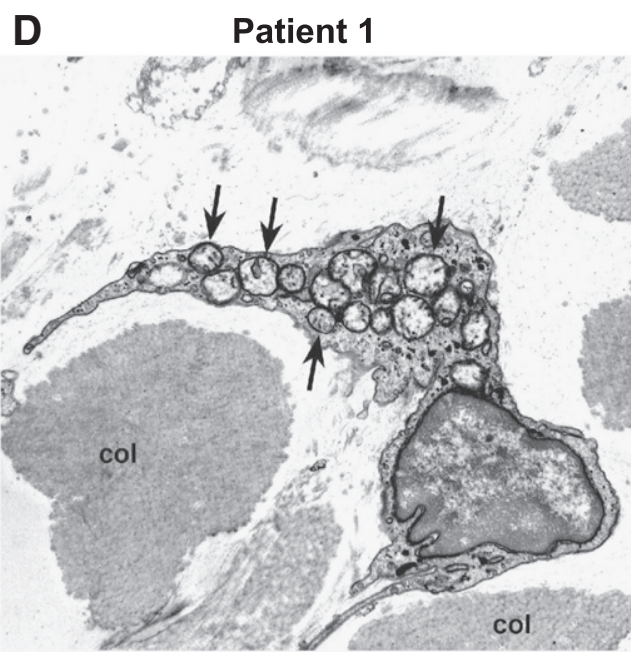

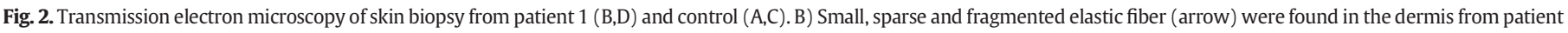

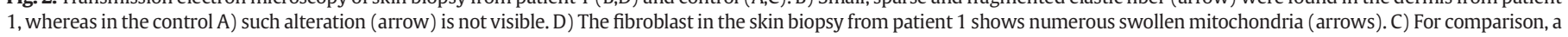
fibroblast of normal skin shows only small mitochondria (arrows). col $=$ collagen fibers. Scale bar A,B $=1 \mu \mathrm{m}, \mathrm{C}=1.5 \mu \mathrm{m}$, and $\mathrm{D}=2 \mu \mathrm{m}$. 
A

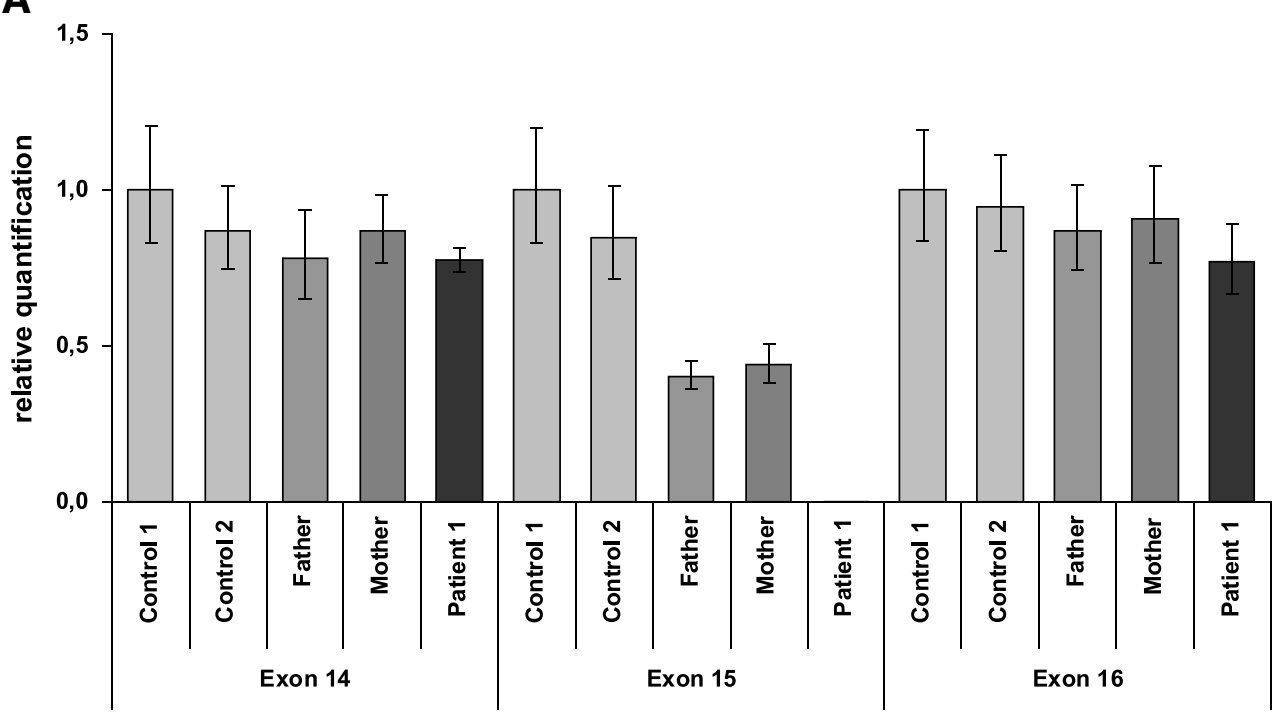

B

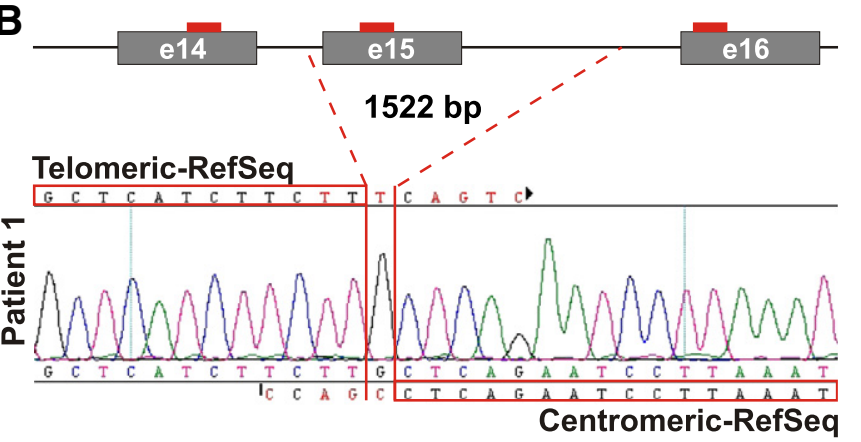

C Exon 17: c.2131delC
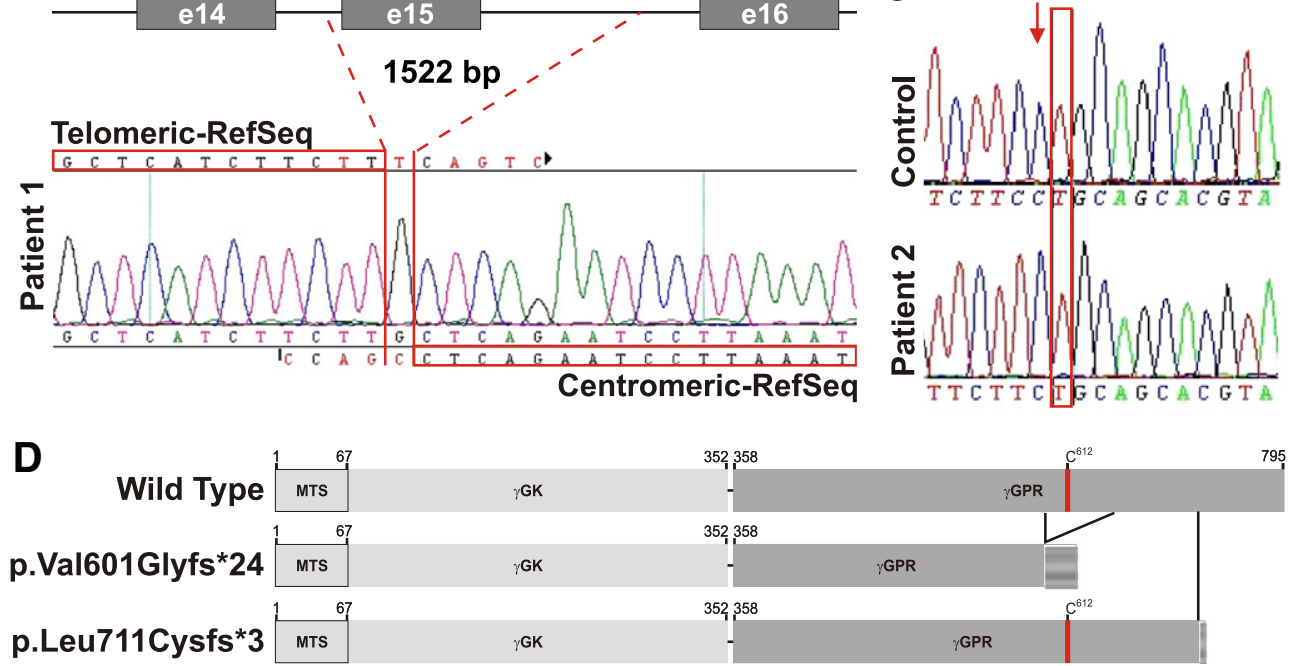

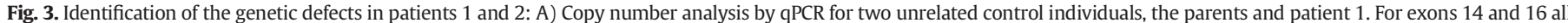

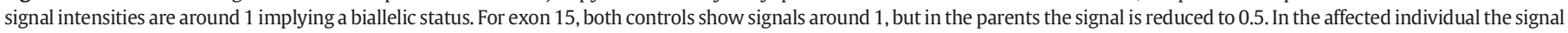

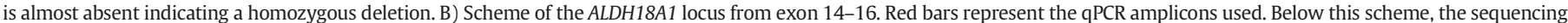

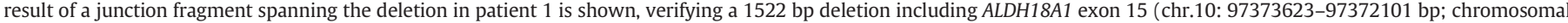

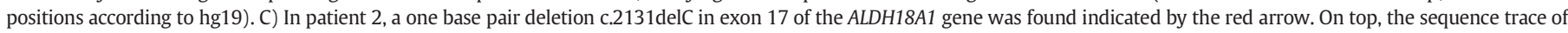

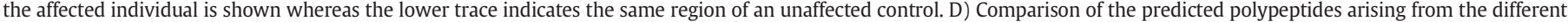
mutated alleles observed in our patients 1 and 2 .

heterozygous carriers. For all unrelated control individuals, the signal intensities remained unchanged (Fig. 3A). For identification of the breakpoints we analyzed different primer combinations within the critical interval. We obtained a junction fragment of $643 \mathrm{bp}$ only for the patient's DNA whereas the control DNAs yielded no product. Sequencing of this fragment finally corroborated a microdeletion of exon 15 and the flanking intronic sequence with a size of 1522 bp (chr.10: 97373623-97372101; chromosomal positions according to hg19) and an insertion of a guanosine (Fig. 3B). The deletion leads to a frameshift with a predicted premature termination codon after 24 additional codons (p.Val601Glyfs*24) (Fig. 3D). In patient 2, a homozygous single-basepair deletion c.2131delC in exon 17 was found (Fig. 3C). This alteration leads to a frameshift and a predicted termination of translation after three additional codons (p.Leu711Cysfs*3) (Fig. 3D).

To further investigate the consequence of the microdeletion observed in patient 1 we performed expression analyses by qPCR on human skin fibroblasts. We found a severe reduction of the ALDH18A1 mRNA level relative to control fibroblasts (Fig. $4 \mathrm{~A}$ ). To study the outcome on the protein level we investigated ALDH18A1 expression by immunoblot. In control fibroblasts we detected a band with the expected size of approximately $85 \mathrm{KDa}$ whereas in the fibroblasts from patient 1 no such band was detectable (Fig. 4B).

\section{Discussion}

Both affected individuals presented with manifestations typical for de Barsy syndrome. Although the etiology of the severe neurocutaneous disorder in the family originally described by de Barsy remains unknown it was shown that de Barsy-like disorders can be due to mutations either in PYCR1 or ALDH18A1 [11,12,14,15]. In our patients, we found clinical features like facial dysmorphism, hypotonia, adducted thumbs and cutis laxa, which are frequently found in progeroid cutis laxa syndromes. 

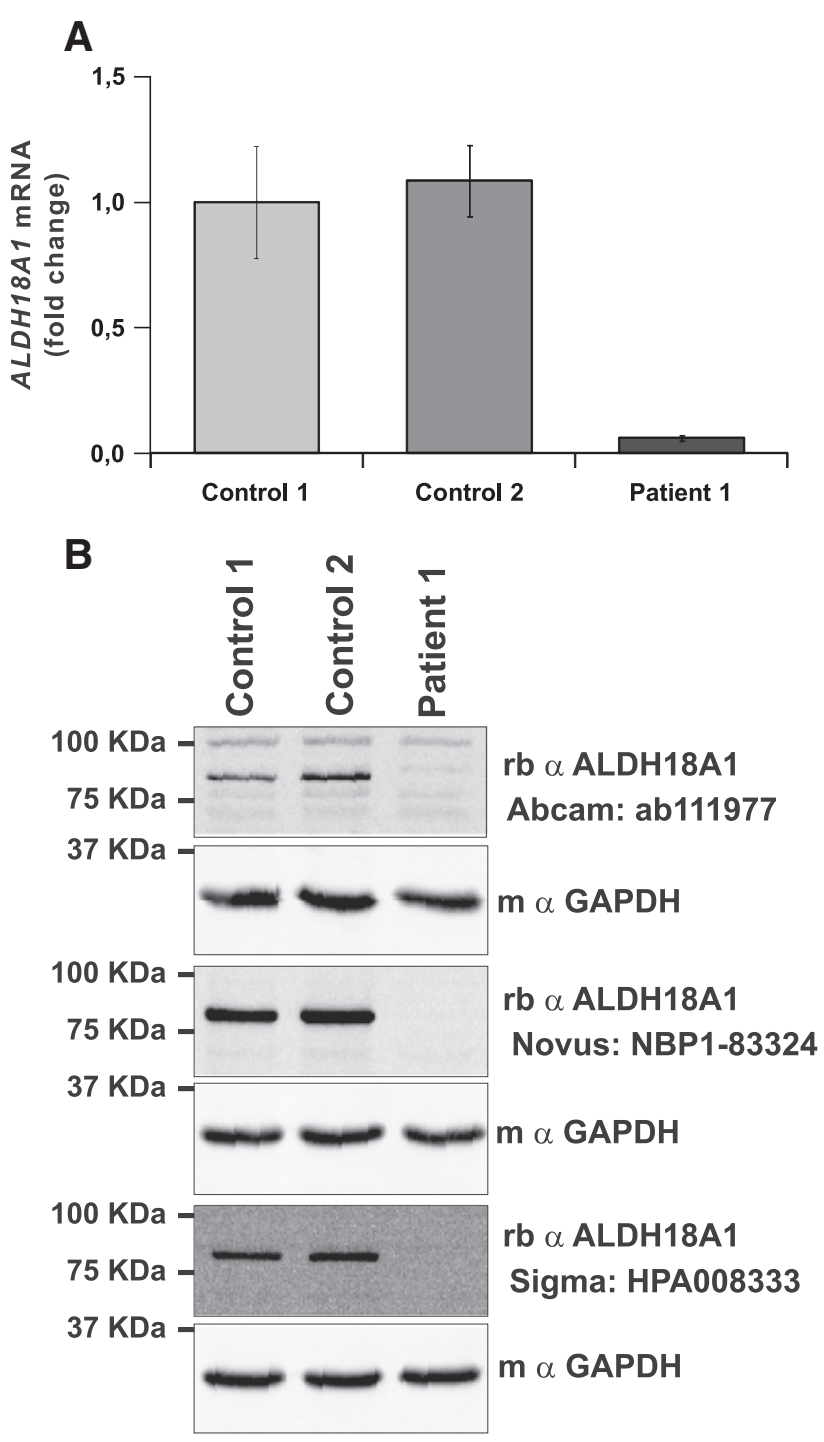

Fig. 4. A) Relative mRNA quantification in fibroblasts from patient 1 relative to control cells shows a strong ALDH18A1 mRNA reduction. B) Representative immunoblot results show that the ALDH18A1 protein is detectable in control fibroblasts whereas it is absent in fibroblasts from patient 1 using three independent antibodies against ALDH18A1. Equal loading was shown analyzing GAPDH as an endogenous control.

However, while osteoporosis or osteopenia is a skeletal feature found in most CL disorders $[6,8,15]$ absence or abnormal shape of distal skeletal elements found in patient 1 is unusual. Clenched or adducted thumbs are a typical sign for ARCL due to PYCR1 or ALDH18A1 mutations. Often the thumbs are subluxated and contractures make surgical intervention necessary. How the special combination of bowing of the first metacarpal and abnormal fifth finger middle phalanges described in patient 1 could be caused by impaired mitochondrial proline synthesis remains elusive.

Surprisingly, we found cardiovascular abnormalities in both patients. Cardiac septal defects are the most common heart malformations and therefore it is unclear whether these defects observed in patient 1 are really related to the genetic alteration. Also the significance of patent ductus arteriosus and persistent superior left vena cava remains unclear. However, the intracranial arterial malformation of patient 2 is previously unreported and had dramatic consequences. In two patients with ALDH18A1-related CL, kinky tortuosity of brain vessels was reported $[16,17]$ demonstrating that in this severe form of CL vessels are especially vulnerable. Therefore, vessels should not only be assessed in ADCL, ARCL1, and ATS that are well known for their arterial malformations $[1,18,19]$, but also in proline biosynthesis pathologies.
This might be a potential diagnostic feature to differentiate between PYCR1- and ALDH18A1-related CL [12,15,20,21].

So far, only 13 affected individuals with ALDH18A1 mutations from seven unrelated families have been described, mainly with missense or splice site mutations $[11,14,16,17,22-24]$. The $~ 1.6 \mathrm{~kb}$ microdeletion identified here is the first mutation of this class found in ALDH18A1related CL. Due to the fact that no microhomologies were found and given the insertion of a guanosine, the underlying mechanism for this genomic rearrangement most likely is a non-homologous end joining (NHEJ), a frequent cause for such non-recurrent rearrangements [25]. Recently, one patient was described carrying a homozygous splice site mutation in ALDH18A1 [17]. In the case presented here, the underlying mechanism is more likely a nonsense-mediated decay, shown by the almost complete absence of the ALDH18A1 mRNA whereas in the case by Skidmore et al. at least the splice product with open reading frame could be partially stable. However, in both patients a total absence of the protein was shown, which contrasts the different severities of the phenotype. In patient 1 no alteration in the coding sequence of PYCR1 was detectable whereas the individual described by Skidmore et al. showed the heterozygous p.Ala110Val substitution, which is also found in the 1000 genome data in a heterozygous state. Our current findings support the idea that this alteration in PYCR1 might modulate the severity due to a possible reduction of PYCR1 enzymatic activity [17]. However, more solid information is necessary to prove this point. Unfortunately, no living cells or tissue was available from patient 2 carrying the p.Leu711Cysfs*3 mutation. The mutated transcript could be either degraded via the NMD pathway or lead to a truncated, but stable protein. The clinical presentation in the two affected individuals varies from a very early lethal to a severe CL phenotype which is in line with phenotype spectrum described in the literature. So far, no clear genotype-phenotype correlation has emerged for ALDH18A1-related cutis laxa.

Since ALDH18A1 is localized to mitochondria where it converts glutamate to proline via pyrrolin-5-carboxylate $[14,26]$ one would imagine that dysfunction of this protein leads to abnormal metabolite concentrations. The investigation of plasma amino acid profiles in patient 1 revealed low levels of citrulline and arginine which is in line with previous observations. Interestingly, only three out of seven studies describing mutations in ALDH18A1 report on those alterations [14, $16,17]$. This might point to a possible compensation through the diet. Alternatively, since both proteins, PYCR1 and ALDH18A1, are restricted to mitochondria, intracellular amino acid concentrations could be altered. Furthermore we found swelling of mitochondria in dermal fibroblasts in the skin biopsy from patient 1 . This might be a sign for mitochondrial dysfunction in vivo. In cultured fibroblasts no such alteration was detectable which could be due to the culture conditions. Depletion of $\mathrm{P} 5 \mathrm{CDH}$, an enzyme of the reverse pathway that converts proline to glutamate, also leads to the swelling of the mitochondria in vivo in drosophila larvae [27]. In plants, intracellular proline was described to have osmoregulatory functions [28]. Whether such a function also exists in human cells has to be further investigated.

The number of individuals with ALDH18A1 mutations is very low [5]. It is possible that a negative selection occurs due to elevated intrauterine lethality induced by biallelic ALDH18A1 mutations. This also might explain the frequent abortions in the mother from patient 2 , which, however, could also be caused by other gene mutations given the consanguinity of the parents. On the other hand low mutation frequency could also be due to low mutation detection rates, e.g. by frequent non-coding mutations or whole gene deletions, which are not detected by standard diagnostic procedures. In the future the detection of possible hidden ALDH18A1 mutations and of alterations in other genes with modifying effects can be further enhanced using next-generation sequencing based approaches.

\section{Conflict of interest}

The authors declare no conflict of interest. 


\section{Web resources}

Online Mendelian Inheritance in Man (OMIM) http://www.ncbi.nlm. gov/Omim/ (for ADCL and ARCL1 and ARCL2A, ARCL2B, DBS, and MACS).

\section{Acknowledgment}

We are grateful to the patients and their families whose cooperation made this study possible. BCL is a postdoctoral research fellow of the Fund for Scientific Research. The study was funded by the Deutsche Forschungsgemeinschaft (KO 2891/1-1) to UK.

\section{References}

[1] B.L. Callewaert, A. Willaert, W.S. Kerstjens-Frederikse, J. De Backer, K. Devriendt, B. Albrecht, M.A. Ramos-Arroyo, M. Doco-Fenzy, R.C. Hennekam, R.E. Pyeritz, O.N. Krogmann, G. Gillessen-kaesbach, E.L. Wakeling, S. Nik-zainal, C. Francannet, P. Mauran, C. Booth, M. Barrow, R. Dekens, B.L. Loeys, P.J. Coucke, A.M. De Paepe, Arterial tortuosity syndrome: clinical and molecular findings in 12 newly identified families, Hum. Mutat. 29 (2008) 150-158.

[2] V. Hucthagowder, N. Sausgruber, K.H. Kim, B. Angle, L.Y. Marmorstein, Z. Urban, Fibulin-4: a novel gene for an autosomal recessive cutis laxa syndrome, Am. J. Hum. Genet. 78 (2006) 1075-1080.

[3] B. Loeys, L. Van Maldergem, G. Mortier, P. Coucke, S. Gerniers, J.M. Naeyaert, A. De Paepe, Homozygosity for a missense mutation in fibulin-5 (FBLN5) results in a severe form of cutis laxa, Hum. Mol. Genet. 11 (2002) 2113-2118.

[4] P.J. Coucke, A. Willaert, M.W. Wessels, B. Callewaert, N. Zoppi, J. De Backer, J.E. Fox, G.M. Mancini, M. Kambouris, R. Gardella, F. Facchetti, P.J. Willems, R. Forsyth, H.C. Dietz, S. Barlati, M. Colombi, B. Loeys, A. De Paepe, Mutations in the facilitative glucose transporter GLUT10 alter angiogenesis and cause arterial tortuosity syndrome, Nat. Genet. 38 (2006) 452-457.

[5] B. Fischer, A. Dimopoulou, J. Egerer, T. Gardeitchik, A. Kidd, D. Jost, H. Kayserili, Y, Alanay, I. Tantcheva-Poor, E. Mangold, C. Daumer-Haas, S. Phadke, R.I. Peirano, J. Heusel, C. Desphande, N. Gupta, A. Nanda, E. Felix, E. Berry-Kravis, M. Kabra, R.A. Wevers, L. van Maldergem, S. Mundlos, E. Morava, U. Kornak, Further characterization of ATP6V0A2-related autosomal recessive cutis laxa, Hum. Genet. 11 (2012) $1761-1773$.

[6] U. Kornak, E. Reynders, A. Dimopoulou, J. van Reeuwijk, B. Fischer, A. Rajab, B. Budde, P. Nurnberg, F. Foulquier, D. Lefeber, Z. Urban, S. Gruenewald, W. Annaert, H.G. Brunner, H. van Bokhoven, R. Wevers, E. Morava, G. Matthijs, L. Van Maldergem, S. Mundlos, Impaired glycosylation and cutis laxa caused by mutations in the vesicular H+-ATPase subunit ATP6V0A2, Nat. Genet. 40 (2008) 32-34.

[7] L. Basel-Vanagaite, O. Sarig, D. Hershkovitz, D. Fuchs-Telem, D. Rapaport, A. Gat, G. Isman, I. Shirazi, M. Shohat, C.D. Enk, E. Birk, J. Kohlhase, U. Matysiak-Scholze, I. Maya, C. Knopf, A. Peffekoven, H.C. Hennies, R. Bergman, M. Horowitz, A. Ishida-Yamamoto, E. Sprecher, RIN2 deficiency results in macrocephaly, alopecia, cutis laxa, and scoliosis: MACS syndrome, Am. J. Hum. Genet. 85 (2009) 254-263.

[8] H.C. Hennies, U. Kornak, H. Zhang, J. Egerer, X. Zhang, W. Seifert, J. Kuhnisch, B. Budde, M. Natebus, F. Brancati, W.R. Wilcox, D. Muller, P.B. Kaplan, A. Rajab, G. Zampino, V. Fodale, B. Dallapiccola, W. Newman, K. Metcalfe, J. Clayton-Smith, M. Tassabehji, B. Steinmann, F.A. Barr, P. Nurnberg, P. Wieacker, S. Mundlos, Gerodermia osteodysplastica is caused by mutations in SCYL1BP1, a Rab-6 interacting golgin, Nat. Genet. 40 (2008) 1410-1412.

[9] A.M. de Barsy, E. Moens, L. Dierckx, Dwarfism, oligophrenia and degeneration of the elastic tissue in skin and cornea. A new syndrome? Helv. Paediatr. Acta 23 (1968) 305-313.

[10] E. Morava, M. Guillard, D.J. Lefeber, R.A. Wevers, Autosomal recessive cutis laxa syndrome revisited, Eur. J. Hum. Genet. 17 (2009) 1099-1110.

[11] L.S. Bicknell, J. Pitt, S. Aftimos, R. Ramadas, M.A. Maw, S.P. Robertson, A missense mutation in ALDH18A1, encoding delta1-pyrroline-5-carboxylate synthase (P5CS), causes an autosomal recessive neurocutaneous syndrome, Eur. J. Hum. Genet. 16 (2008) 1176-1186

[12] B. Reversade, N. Escande-Beillard, A. Dimopoulou, B. Fischer, S.C. Chng, Y. Li, M. Shboul, P.Y. Tham, H. Kayserili, L. Al-Gazali, M. Shahwan, F. Brancati, H. Lee, B.D. O'Connor, M. Schmidt-von Kegler, B. Merriman, S.F. Nelson, A. Masri, F. Alkazaleh, D. Guerra, P. Ferrari, A. Nanda, A. Rajab, D. Markie, M. Gray, J. Nelson, A. Grix, A. Sommer, R. Savarirayan, A.R. Janecke, E. Steichen, D. Sillence, I. Hausser, B. Budde, G. Nurnberg, P. Nurnberg, P. Seemann, D. Kunkel, G. Zambruno, B. Dallapiccola, M. Schuelke, S. Robertson, H. Hamamy, B. Wollnik, L. Van Maldergem, S. Mundlos, U.
Kornak, Mutations in PYCR1 cause cutis laxa with progeroid features, Nat. Genet. 41 (2009) 1016-1021.

[13] C.E. Ott, G. Leschik, F. Trotier, L. Brueton, H.G. Brunner, W. Brussel, E. GuillenNavarro, C. Haase, J. Kohlhase, D. Kotzot, A. Lane, M.A. Lee-Kirsch, S. Morlot, M.E. Simon, E. Steichen-Gersdorf, D.H. Tegay, H. Peters, S. Mundlos, E. Klopocki, Deletions of the RUNX2 gene are present in about $10 \%$ of individuals with cleidocranial dysplasia, Hum. Mutat. 31 (2010) E1587-E1593.

[14] M.R. Baumgartner, C.A. Hu, S. Almashanu, G. Steel, C. Obie, B. Aral, D. Rabier, P. Kamoun, J.M. Saudubray, D. Valle, Hyperammonemia with reduced ornithine, citrulline, arginine and proline: a new inborn error caused by a mutation in the gene encoding delta(1)-pyrroline-5-carboxylate synthase, Hum. Mol. Genet. 9 (2000) 2853-2858.

[15] A. Dimopoulou, B. Fischer, T. Gardeitchik, P. Schroter, H. Kayserili, C. Schlack, Y. Li, J M. Brum, I. Barisic, M. Castori, C. Spaich, E. Fletcher, Z. Mahayri, M. Bhat, K.M. Girisha, K. Lachlan, D. Johnson, S. Phadke, N. Gupta, M. Simandlova, M. Kabra, A. David, L. Nijtmans, D. Chitayat, B. Tuysuz, F. Brancati, S. Mundlos, L. Van Maldergem, E. Morava, B. Wollnik, U. Kornak, Genotype-phenotype spectrum of PYCR1-related autosomal recessive cutis laxa, Mol. Genet. Metab. 3 (2013) 352-361.

[16] D. Martinelli, J. Haberle, V. Rubio, C. Giunta, I. Hausser, R. Carrozzo, N. Gougeard, C. Marco-Marin, B.M. Goffredo, M.C. Meschini, E. Bevivino, S. Boenzi, G.S. Colafati, F. Brancati, M.R. Baumgartner, C. Dionisi-Vici, Understanding pyrroline-5-carboxylate synthetase deficiency: clinical, molecular, functional, and expression studies, structure-based analysis, and novel therapy with arginine, J. Inherit. Metab. Dis. 5 (2012) 761-776.

[17] D.L. Skidmore, D. Chitayat, T. Morgan, A. Hinek, B. Fischer, A. Dimopoulou, G. Somers W. Halliday, S. Blaser, Y. Diambomba, E.G. Lemire, U. Kornak, S.P. Robertson, Further expansion of the phenotypic spectrum associated with mutations in ALDH18A1, encoding delta(1)-pyrroline-5-carboxylate synthase (P5CS), Am. J. Med. Genet. A 155A (2011) 1848-1856.

[18] B. Callewaert, C.T. Su, T. Van Damme, P. Vlummens, F. Malfait, O. Vanakker, B. Schulz M. Mac Neal, E.C. Davis, J.G. Lee, A. Salhi, S. Unger, K. Heimdal, S. De Almeida, U. Kornak, H. Gaspar, J.L. Bresson, K. Prescott, M.E. Gosendi, S. Mansour, G.E. Pierard, S. Madan-Khetarpal, F.C. Sciurba, S. Symoens, P.J. Coucke, L. Van Maldergem, Z. Urban, A. De Paepe, Comprehensive clinical and molecular analysis of 12 families with type 1 recessive cutis laxa, Hum. Mutat. 34 (2013) 111-121.

[19] B. Callewaert, M. Renard, V. Hucthagowder, B. Albrecht, I. Hausser, E. Blair, C. Dias, A Albino, H. Wachi, F. Sato, R.P. Mecham, B. Loeys, P.J. Coucke, A. De Paepe, Z. Urban, New insights into the pathogenesis of autosomal-dominant cutis laxa with report of five ELN mutations, Hum. Mutat. 32 (2011) 445-455.

[20] R. Kretz, B. Bozorgmehr, M.H. Kariminejad, M. Rohrbach, I. Hausser, A. Baumer, M Baumgartner, C. Giunta, A. Kariminejad, J. Haberle, Defect in proline synthesis: pyrroline-5-carboxylate reductase 1 deficiency leads to a complex clinical phenotype with collagen and elastin abnormalities, J. Inherit. Metab. Dis. 34 (2011) 731-739.

[21] Y. Yildirim, A. Tolun, B. Tuysuz, The phenotype caused by PYCR1 mutations corresponds to geroderma osteodysplasticum rather than autosomal recessive cutis laxa type 2, Am. J. Med. Genet. A 155A (2011) 134-140.

[22] S. Zampatti, M. Castori, B. Fischer, P. Ferrari, L. Garavelli, C. Dionisi-Vici, E. Agolini, A. Wischmeijer, E. Morava, G. Novelli, J. Haberle, U. Kornak, F. Brancati, De Barsy Syndrome: a genetically heterogeneous autosomal recessive cutis laxa syndrome related to P5CS and PYCR1 dysfunction, Am. J. Med. Genet. A 158A (2011) 927-931.

[23] M. Handley, A. Mégarbané, A. Meynert, S. Brown, E. Freyer, M. Taylor, I. Jackson, I. Aligianis, Loss of ALDH18A1 function is associated with a cellular lipid droplet phenotype suggesting a link between autosomal recessive cutis laxa type $3 \mathrm{~A}$ and Warburg Micro syndrome, Mol. Genet. Genomics Med. (2014) [Epub ahead of print].

[24] D.F. Wolthuis, E. van Asbeck, M. Mohamed, T. Gardeitchik, E. R. Lim-Melia, R.A. Wevers, E. Morava, Cutis laxa, fat pads and retinopathy due to ALDH18A1 mutation and review of the literature, Eur. J. Paediatr. Neurol. (2014) [Epub ahead of print].

[25] W. Gu, F. Zhang, J.R. Lupski, Mechanisms for human genomic rearrangements, Pathogenetics 1 (2008) 4

[26] M.R. Baumgartner, D. Rabier, M.C. Nassogne, J.L. Dufier, J.P. Padovani, P. Kamoun, D. Valle, J.M. Saudubray, Delta1-pyrroline-5-carboxylate synthase deficiency: neurodegeneration, cataracts and connective tissue manifestations combined with hyperammonaemia and reduced ornithine, citrulline, arginine and proline, Eur. J. Pediatr. 164 (2005) 31-36.

[27] F. He, PJ. DiMario, Drosophila delta-1-pyrroline-5-carboxylate dehydrogenase ( $\mathrm{P} 5 \mathrm{CDh}$ ) is required for proline breakdown and mitochondrial integrity-establishing a fly model for human type II hyperprolinemia, Mitochondrion 11 (2011) 397-404.

[28] D. Rhodes, S. Handa, R.A. Bressan, Metabolic changes associated with adaptation of plant cells to water stress, Plant Physiol. 82 (1986) 890-903. 